\title{
Improving failure analysis efficiency by combining FTA and FMEA in a recursive manner
}

\author{
J.F.W. Peeters ${ }^{a}$, R.J.I. Basten ${ }^{b, *}$, T. Tinga ${ }^{c, d}$ \\ a Additive Industries B.V., Leidingstraat 27, 5617 AJ, Eindhoven, The Netherlands \\ ${ }^{\mathrm{b}}$ Eindhoven University of Technology, School of Industrial Engineering, P.O. Box 513, 5600 MB, Eindhoven, The Netherlands \\ ${ }^{\mathrm{c}}$ Netherlands Defense Academy, P.O. Box 10000, 1780 CA, Den Helder, The Netherlands \\ ${ }^{\mathrm{d}}$ University of Twente, Faculty of Engineering Technology, P.O. Box 217, 7500 AE, Enschede, The Netherlands
}

\section{A R T I C L E I N F O}

\section{Keywords:}

FMEA

FTA

Failure analysis

Additive manufacturing system

\begin{abstract}
A B S T R A C T
When designing a maintenance programme for a capital good, especially a new one, it is of key importance to accurately understand its failure behaviour. Failure mode and effects analysis (FMEA) and fault tree analysis (FTA) are two commonly used methods for failure analysis. FMEA is a bottom-up method that is less structured and requires more expert knowledge than FTA, which is a top-down method. Both methods are time-consuming when applied thoroughly, which is why in many cases, they are not applied at all. We propose a method in which both are used in a recursive manner: First, a system level FTA is performed, which results in a set of failure modes. Using FMEA, the criticality of the failure modes is assessed in order to select only the critical system level failure modes. For each of those, a function level FTA is performed, followed by an FMEA. Finally, a component level FTA and FMEA are performed on the critical function level failure modes. We apply our method to a recently developed additive manufacturing system for metal printing, the MetalFAB1 of Additive Industries (AI), and find that the engineers at AI consider the method to be efficient and effective.
\end{abstract}

(c) 2017 Elsevier Ltd. All rights reserved.

\section{Introduction}

Advanced capital goods are expensive, technologically advanced systems that are used in the primary processes of their users. When such a system fails, it is thus of utmost importance that the failure is corrected quickly. It is even better to perform preventive maintenance before a failure occurs, which requires good predictions of the failure behaviour of the system. Failure analysis may be difficult when multiple systems have been in use for a while, but is even more difficult for new systems that have not been installed, or only recently.

There exist two methods that are commonly used for failure analysis. The first method is failure mode and effects analysis (FMEA). It is a bottom-up method, starting at the component level, that is used to find failure modes and map their effects. By adding a criticality analysis, the qualitative FMEA can be extended to a quantitative FMECA (failure mode, effects, and criticality analysis). The second method for failure analysis is fault tree analysis (FTA), which is a top-down method that is used to map the relationships between events such as sub-system failures and their causes. Both methods are very time-consuming to apply thoroughly, which is why that is often not done. However, that means that possible failure modes may not be identified. Section 2 will provide more details on the methods and their shortcomings.

In this paper, we propose a structured method that does not take too much time (i.e., is efficient), while it does enable its users to find all relevant failure modes (i.e., is effective). The key idea is to apply FTA and FMEA in a recursive manner: First, a system level FTA is performed, which results in a set of failure modes. Using FMEA, the criticality of the failure modes is assessed in order to select only the critical system level failure modes. For each of those, a function level FTA is performed, followed by an FMEA on those failure modes. Finally, a component level FTA and FMEA are performed on the critical function level failure modes. The method is applied to an additive manufacturing system for metal printing (i.e., a 3D printer) that was recently developed by Additive Industries (AI): the MetalFAB1. Fig. 4 in Section 4 shows the most popular configuration of this system. Engineers at AI find the method to be efficient and effective indeed.

The remainder of the paper is structured as follows. Section 2 discusses the literature on methods for failure analysis and concludes, in Section 2.5, with a more detailed explanation of the contribution of our paper. Next, Section 3 explains the recursive method that we propose.

\footnotetext{
* Corresponding author.

E-mail addresses: j.peeters@additiveindustries.com (J.F.W. Peeters), r.j.i.basten@tue.nl (R.J.I. Basten), t.tinga@utwente.nl (T. Tinga).
} 


\begin{tabular}{|c|c|c|c|c|c|c|c|c|c|}
\hline \multicolumn{6}{|c|}{ Failure mode effects and criticallity analysis } & \multirow{2}{*}{\multicolumn{4}{|c|}{$\frac{\text { date }}{\text { Modules }}$}} \\
\hline \multirow{2}{*}{\multicolumn{2}{|c|}{$\begin{array}{c}\text { Machine function } \\
\text { System }\end{array}$}} & \multirow{2}{*}{\multicolumn{2}{|c|}{$\begin{array}{c}\text { Module } \\
\text { sYs }\end{array}$}} & \multirow{2}{*}{\multicolumn{2}{|c|}{$\begin{array}{l}\text { Attendees } \\
\text { Johnny Peeters }\end{array}$}} & & & & \\
\hline & & & & & & \multicolumn{4}{|c|}{ NAME } \\
\hline \multirow[t]{2}{*}{ Function } & \multirow[t]{2}{*}{ Module } & $\begin{array}{l}\text { Failure } \\
\text { Mode }\end{array}$ & \multicolumn{2}{|c|}{ Possible Failure Effects } & s & $\begin{array}{c}\text { Failure } \\
\text { Causes } \\
\end{array}$ & 0 & $\begin{array}{l}\text { Failure detection } \\
\text { method }\end{array}$ & D D RPN \\
\hline & & & Local effect & End effect & & & & & \\
\hline $\begin{array}{l}\text { System } \\
\text { unction }\end{array}$ & & $\begin{array}{l}\text { Failure } \\
\text { mode } 1\end{array}$ & $\begin{array}{l}\text { Local effect of } \\
\text { failure mode } 1\end{array}$ & $\begin{array}{l}\text { End effect of } \\
\text { failure mode } 1\end{array}$ & & $\begin{array}{l}\text { Cause of } \\
\text { failure mode }\end{array}$ & & $\begin{array}{l}\text { Detection method } \\
\text { of fallure mode } 1\end{array}$ & $\begin{array}{l}=S \times \\
0 \times D\end{array}$ \\
\hline
\end{tabular}

Fig. 1. FMEA/FMECA spreadsheet.

The method is applied to the case of the MetalFAB1 in Section 4. Finally, Section 5 concludes.

\section{Literature}

In this section, two commonly used methods for failure analysis are introduced: FMEA (Failure Mode and Effects Analysis) in Section 2.1 and FTA (Fault Tree Analysis) in Section 2.2. Subsequently, a review on published work on combinations of FTA and FMEA is discussed in Section 2.3. Section 2.4 discusses the shortcomings of existing methods, and, finally, Section 2.5 describes the position of this paper and its contribution.

\subsection{Failure mode and effects analysis}

FMEA (Failure Mode and Effects Analysis) is a systematic method to map failure modes, effects and causes of technical systems [1]. It is an inductive and bottom-up method, since the analysis starts at the component level where the possible component failure modes are identified and it is examined what the consequences are on a higher level. Usually, the FMEA is carried out with a diverse team of people with various backgrounds (e.g., mechanical design, software, operations, maintenance) since this increases the probability that all possible failures are identified and the effects properly estimated [2]. The FMEA can be extended to a FMECA (Failure Mode, Effects and Criticality Analysis) by adding a criticality analysis. In this way, the purely qualitative FMEA can be made more quantitative. In the FMECA, the criticality of each failure mode is quantified by the risk priority number (RPN). The RPN is discussed in more detail in Section 3.2.2. The results of an FMEA/FMECA are usually recorded in a table, such as the table shown in Fig. 1. Standards are available that provide guidelines for performing a FMEA/FMECA, see, for example, [3] or [4]. For convenience, in this paper the term FMEA will be used for the generic method, regardless whether it does or does not contain a criticality analysis (FMECA or FMEA, respectively).

\subsection{Fault Tree Analysis}

Fault Tree Analysis (FTA) is an alternative method to investigate failure behavior. A fault tree is a logic diagram that represents the relationships between an event (typically a system failure) and the causes of the event (typically component failures). It uses logic gates and events to model how the component states relate to the state of the system as a whole. The commonly used logic gates in FTA are: the (1) OR-gate, (2) AND-gate and (3) inhibit or conditional gate. The commonly used eventtypes in FTA are the (1) top or intermediate event, (2) basic event, (3) diamond or undeveloped event and (4) conditional event. A detailed description of the symbols used in FTA can be found in, for example [5]. Furthermore, the purely qualitative FTA can be extended into a quantitative FTA [1] by adding quantitative information of component reliability (e.g., failure rates). Such a quantitative FTA can be used to determine the reliability of the system using boolean algebra [6]. Ruijters and Stoelinga [7] give an extensive overview of (mainly quantitative) FTAs.

\subsection{Combination of FTA and FMEA}

FTA and FMEA can be combined in a failure analysis to gain the individual benefits of both approaches. Two options can be identified:
(1) perform both an FMEA as well as an FTA separately or (2) use a mixed approach.

Concerning the first option, some authors argue to use FTA and FMEA complementary to each other [1]. For instance, Bertsche [1] states that this may expand the number of failure modes found due to the different starting points of both methods: bottom-up in FMEA versus topdown in FTA. However, performing both analyses would be rather time consuming and may lead to a loss of focus on the most critical parts of the system, which the failure analysis typically aims to identify.

Alternatively, one can decide to use a mixed approach that is a combination of FTA and FMEA. This has been proposed by some authors. Yu et al. [8] propose a mixed approach in which the FMEA is guided by an FTA. In their proposed approach, the analysis starts with the definition of a system failure event and the construction of a fault tree for a particular system as a whole. Subsequently, the minimal cut set of the fault tree is determined to identify the basic events. Finally, each basic event is analyzed further with FMEA to identify the underlying failure modes of each component. Bluvband et al. [9] propose another combined method of FTA and FMEA which is called Bouncing Failure Analysis (BFA). In BFA, the analysis starts with the definition of the top failure events on the system level, which are called end effects. Next, a fault tree is constructed to find all possible failure modes on the component level. Subsequently, each identified component failure mode is evaluated in an FMEA and the direct effects to the end effects (called single-point effects) are investigated. Then, the higher order interactions (called double-point, triple-point, etc.) are investigated with an interaction matrix. This allows the analyst to "bounce" back to FTA and create the corresponding fault tree. In this way, the analyst has two representations of the failure behavior. Finally, Han et al. [10] propose a combined analysis method of FMEA and FTA for the safety analysis of critical software. The idea of this approach is similar to the approaches reported in [8] and [9], but is specifically tailored for the analysis of software. In this paper, the focus is on failure analysis of hardware systems, which is of a different nature, and therefore the work of Han et al. is less relevant for the present work.

Related to the discussion of mixed approaches of FMEA and FTA is streamlined Reliability-Centered Maintenance (RCM) [11,12]. Streamlined RCM is a less rigorous derivative of the original RCM process of Nowlan and Heap [13]. The original RCM is defined as a process used to determine what must be done to ensure that a system continues to do what it should do. Since the complete RCM process is comprehensive, there are attemps to "streamline" the RCM process. Moubray [11] states that a way to streamline the RCM process is to analyse only the critical functions or failures of a system. However, he argues that it is difficult to determine which functions or failures are critical and that gained benefits do not outweigh the extra effort required to distinguish between critical and non-critical failures. There are also ways to extend the RCM, see, for example, [14].

\subsection{Shortcomings of existing methods}

For the failure analysis of a newly developed and highly complex system, the most obvious choice would be to use either a standard FMEA or a standard FTA for the complete analysis. However, that would lead to the following difficulties.

We start with discussing the use of a FMEA only. FMEA is an inductive and non-structured approach to identify failure modes and design weaknesses. Furthermore, a quantitative measure of risk (the risk priority number, see Sections 2.1 and 3.2.2) can be incorporated in the analysis. FMEA is most effective when it is used in sessions with a diverse team and when the team members have experience with the operation of the machine [1]. For the analysis of a typical new and complex system this offers a number of challenges. Firstly, the failure behaviour of such a new system is not known from practice. Secondly, this type of system is typically large and complex. This means that in a non-structured approach, it may be difficult to define a starting point and maintain a 
focus on the most critical failures. In addition, the bottom-up approach of FMEA may make it even more difficult to determine "where to search for failure modes" when there is a lack of practical experience. Lastly, when FMEA is applied to a complete system it may be hard to achieve enough depth of analysis to get a full understanding of the failure behaviour.

Also the deductive and structured FTA can be used as single method to analyze the failure behavior. Although FTA has some advantages over FMEA, it still has some drawbacks when analyzing complex systems. Firstly, the structured approach of FTA is an advantage when a completely new system is analyzed and little practical experience with system failures from the field is available. Due to the structured and deductive reasoning implied in FTA, it relies less on practical experience of the expert than FMEA does. In addition, FTA can also be considered to be a more rigorous approach due to the step-by-step reasoning. Secondly, FTA is a graphical method that is easier to interpret and to identify interrelations compared to FMEA and forces the analyst to decompose the system. Thirdly, the complexity of the system under analysis implies that it would be difficult to perform an FMEA over the complete system with enough depth of analysis [8]. This is also the case for FTA, but the analyst may choose to only go deeper into specific parts or branches of the fault tree, which makes FTA a more controllable approach. If the analyst decides to not go deeper into a specific branch of the fault tree, he or she can use the diamond event that represents that the branch is not analysed further. However, there are no strict guidelines to decide on the use of a diamond event and this decision is often being made arbitrarily or based on expert judgement. Our method ensures that this decision is made in a structured way.

Alternatively, a mixed approach of FMEA and FTA can be applied. As discussed in Section 2.3, several authors propose a mixed approach of FTA and FMEA. Both Yu et al. [8] and Bluvband et al. [9] propose to start the analysis with an FTA followed by an FMEA. Yu et al. aim to decompose the failure analysis with FTA into different main components to perform an FMEA on. Bluvband et al. aim to create both a fault tree as well as the FMEA table in one failure analysis. These ideas are interesting, because they enable to combine the strengths of both methods.

However, these methods also possess shortcomings. First, both methods distinguish only between two levels: (1) system level and (2) component level. In large complex systems, one may identify more than two levels. Such systems typically have a layered architecture, which consists of multiple (sub)modules and (sub)assemblies. For such systems application of the methods quickly becomes infeasible. Second, the importance of the level of detail in a failure analysis is not treated with much emphasis, in other words, the methods provide no guidance in what is considered in the analysis and when the analysis is sufficiently detailed. Third, the methods do not possess a feature to focus the analysis on the most critical elements of a machine. Especially for complex systems, the analysis may be comprehensive. For this kind of analyses, one may want to focus on the most critical parts of the system and allocate more attention to these parts. This is related to the efficiency of the analysis, so the required effort for the analysis versus the gained knowledge of failure behaviour.

\subsection{Contribution}

This paper proposes a novel approach for a failure analysis that uses FTA and FMEA multiple times in a recursive way. The approach is discussed in more detail in Section 3.

The contribution of this paper is twofold. First, the proposed method explicitly takes into account the importance of the level of detail in a failure analysis. It provides guidance on what is considered in the analysis and when the analysis is sufficiently detailed on a certain level. This is defined by the level of analysis. Second, the proposed methodology improves the efficiency of a failure analysis for new complex systems by decreasing the required effort for the analysis while still gaining knowledge of the most critical failure behaviour.

\section{Recursive FTA-FMEA}

In this paper, a new approach is presented that uses FTA and FMEA multiple times in a recursive way. First, the global structure of the methodology is described in terms of the level of analysis (Section 3.1) and stages in a failure analysis (Section 3.2). Subsequently, the analysis procedure is described in Section 3.3. The key idea is to perform an FTA to identify failure modes and then an FMEA to assess the criticality of each failure mode top-down at three different levels. Fig. 2 gives an overview of the method.

\subsection{Level of analysis}

Consider a complex system $S$ with $n$ different functions $F_{i}$. For the system to perform a function $F_{i}$, it uses some of the $m$ components in the system. As a result, each function $F_{i}$ cascades into 1 up to $m$ different components $C_{j}$. See Fig. 3 for a schematic representation of this architecture.

To analyze the failure behavior of system $S$, the failure analysis can be related to the different levels in the system, resulting in three levels of analysis: (1) system level, (2) function level and (3) component level. The level of analysis describes the scope and depth of each part of the analysis, in other words, it describes what is considered in the analysis and when the analysis is sufficiently detailed. Decoupling the analyses at the different levels in this manner is advantageous, since it ensures that each part of the analysis has a clear scope and goal which improves the effectiveness of the analysis [1].

In the method presented in this paper, the level of analysis changes during the failure analysis procedure. Starting on the system level, the system as a whole is considered and the analyst only considers failure modes of the next level of the system, the functions. More specifically, the aim is to analyse functional failure modes. A functional failure mode is defined as a failure mode that describes a failure of a major system function. Then, on the function level, the analysis shifts one level deeper. In this part of the analysis, the analyst again only considers failures of the next level of the system (component level). The analysis now focuses on component failure modes, which describe failures of specific components of system $S$. Finally, the analysis is targeted on individual components. Now the analyst considers the individual failure modes in a component, which shifts the depth of analysis to failure mechanisms. Failure mechanisms describe the physical mechanisms underlying the failures of parts, components or structures [2]. Table 1 presents an overview of the scope and depth of analysis for each level of analysis.

\subsection{Stages in a failure analysis}

At each level of analysis, the analysis can be split into two different stages: (1) identification of failure modes and (2) assessment of criticality, discussed in Sections 3.2.1 and 3.2.2, respectively. In the first stage, the aim is to identify faults and failure modes in the system. In the second stage, the aim is to analyse the priority (in terms of criticality) of the identified faults and failure modes. In each level of analysis (see Section 3.1), both analysis stages (1) and (2) are present, leading to two types of analyses on each level. Decoupling between the different levels in combination with the quantification of the criticality at each level, allows the overall analysis to prioritize and focus the analysis on the most critical failures.

\subsubsection{Identification of failure modes}

The first stage of the analysis is the identification of failure modes. We argue that, in our proposed methodology, FTA is preferred over FMEA in this stage, because of its (1) structure, (2) reduced dependency on user experience and (3) rigour. (See Section 2.4 for a discussion of these advantages.) The choice for FTA instead of FMEA in this stage has also two plausible negative consequences. First, a negative consequence of the rigour in FTA is that it can be time consuming. However, 


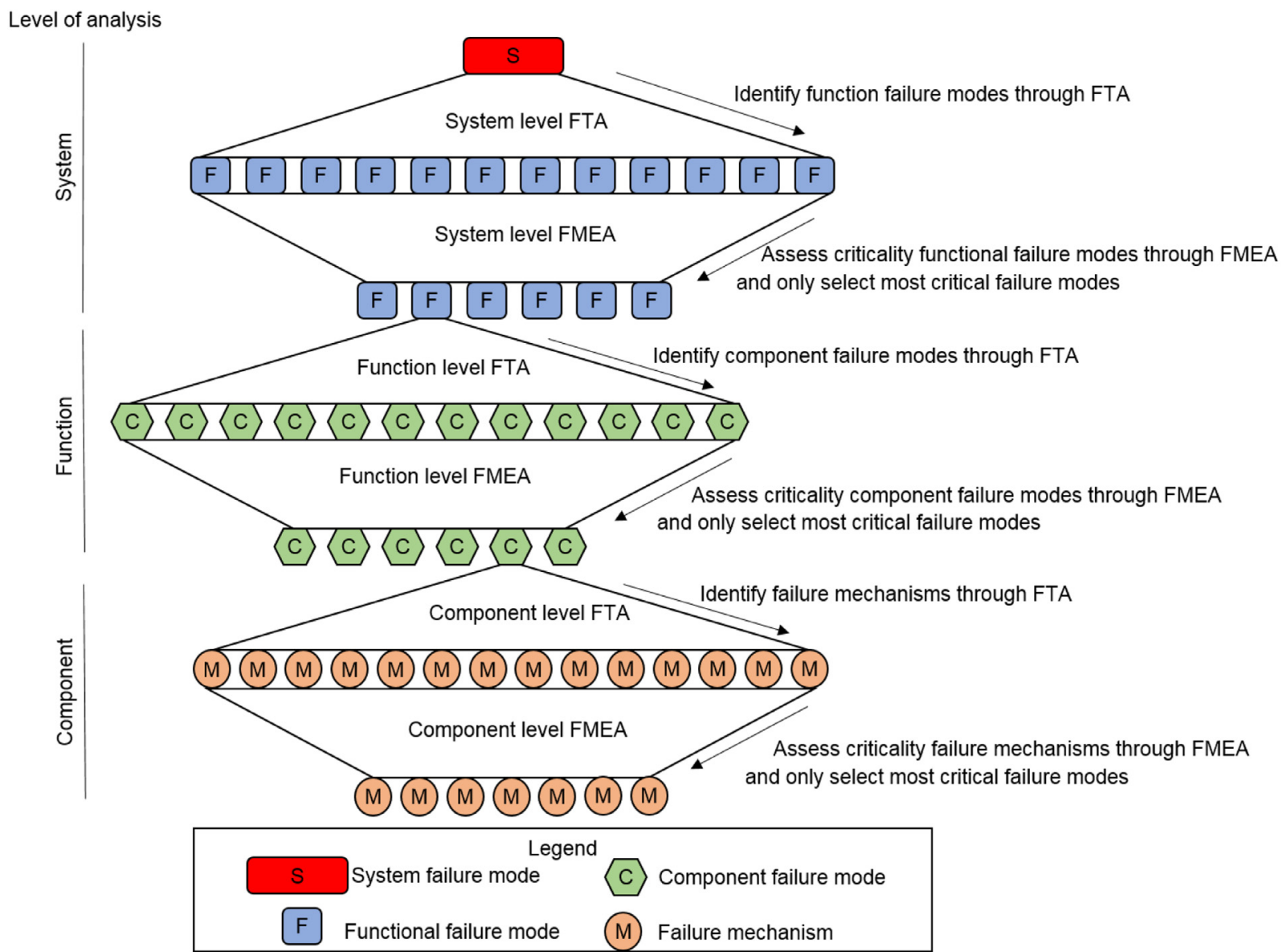

Fig. 2. Recursive FTA-FMEA.

Table 1

Level of analysis.

\begin{tabular}{lll}
\hline Level of analysis & Scope & Depth of analysis \\
\hline System & System and underlying functions & Functional failure modes \\
Function & Function and underlying components & Component failure modes \\
Component & Component and underlying failure mechanisms & Failure mechanisms \\
\hline
\end{tabular}

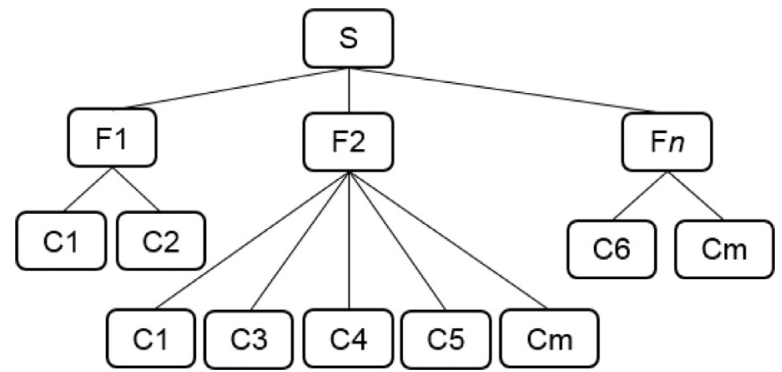

Fig. 3. System definition

in the proposed methodology this is tackled by the decoupling into three parts with each a limited scope; only perform a (detailed) FTA for those functions (in the function level) and those components (in the component level) that have a high criticality. Second, FTA lacks of a method for assessment of criticality; an FMEA can be easily extended to an FMECA whereas the FTA does not possess such a feature. This issue is tackled in our approach by performing a separate assessment of criticality after each FTA.

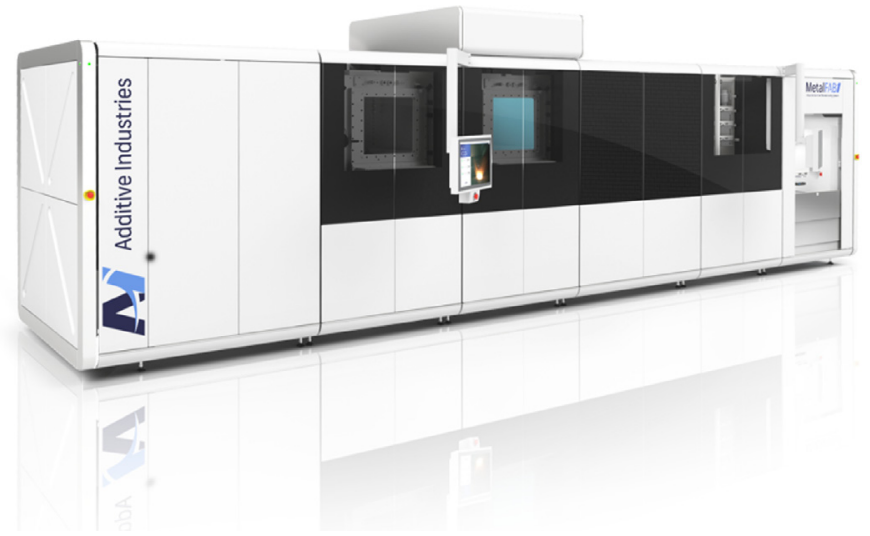

Fig. 4. MetalFAB1.

\subsubsection{Assessment of criticality}

The second stage of the analysis aims to prioritize the identified failure modes in terms of criticality. For this assessment of criticality of the failure modes in each of the different levels, FMEA is the preferred in- 
strument. In FMEA a detailed approach is found by means of the Risk Priority Number (RPN). The RPN is the product of three indicators (typically rated from 1 to 10): (1) a severity indicator (S), (2) an occurrence indicator (O) and (3) a detection indicator (D) of a failure. The severity of a failure refers to the seriousness of the effect or impact of a certain failure and is rated from low impact to very high impact. The occurrence indicator of a failure refers to its failure frequency and is rated from very unlikely to occur to almost inevitable. Finally, the detectability indicator refers to the likelihood that the failure is not detected before it induces major subsequent effects (e.g., by means of process controls, procedures or operator detectability). The detectability indicator is rated from almost sure detection to almost sure non-detection.

Notice that we propose a top-down approach, which implies that when determining $\mathrm{S}, \mathrm{O}$ and $\mathrm{D}$ at a certain level, the values on these indicators at the next lower level are not incorporated explicitly. To be able to do the latter, we would have to use a bottom-up approach in which we would analyse the complete tree with all branches, which is opposing the aim of our method.

A drawback of the method of criticality assessment in FMEA may be the slightly naive way of calculating the RPN. In traditional FMEA, the RPN is calculated by multiplying the three indices for severity, occurrence and detectability, which implies that each index is equally important. See Liu et al. [15] for an overview of the criticism on the usage of the RPN and possible alternatives. One alternative can be to use a weighted RPN calculation method [16] or a fuzzy method [17], which allow to incorporate a weight factor for each index. Alternatively, a more advanced method can be used to prioritize among failure modes, for example by means of a multiple-criteria decision-making method. The Analytical Hierarchy Process (AHP) is such a method that is especially suitable for complex decisions that involve the comparison of decision elements $[18,19]$. In AHP, the decision maker starts with an overall goal, the general objective. That objective can be reached if one tries to maximize a set of different criteria as much as possible. Moreover, for reaching the objective, there are different alternatives considered. This can be captured schematically in a decision hierarchy. Subsequently, based on pairwise comparisons, one can determine weights of each criterium and finally an overall score for each alternative. See [19] for a detailed discussion on AHP. AHP seems to be a good alternative to assess the criticality of failure modes and to select the critical failure modes that should be analysed further. However, in AHP, all alternatives (in this context failures modes) are compared in a pairwise manner. In case there are quite many failure modes, the AHP will be time consuming. Moreover, the definition of the different criteria used in the decision process may be debatable and difficult. In conclusion, we argue that a relatively simple and proven approach by means of FMEA with the simple RPN (in which $R P N=S \cdot O \cdot D$ ) is an effective approach in our methodology.

\subsection{Analysis procedure}

Based on the decoupling of the analysis in terms of level of analysis and stages of analysis, a novel failure analysis methodology has been built that integrates the principles of FTA and FMEA. We have already seen a schematic overview of this failure analysis methodology in Fig. 2. Following this scheme, the details of the proposed method are discussed for each of the levels in Sections 3.3.1-3.3.3.

\subsubsection{System level}

The recursive FTA-FMEA starts with an analysis on the system level.

In the first stage, an FTA on the system level is performed to identify the principal functional failure modes of the system functions, defined as functional failure modes. The initial starting point for a system level FTA is to define a top event for a failure of the system as a whole (system failure mode). This top event has to be defined clearly, because it determines the effectiveness of the whole analysis. The scope of the analysis is the whole system. The depth of analysis is down to the level of functional failure modes. More specifically, a system functional failure mode is defined as a failure of a major system function for which its effects, causes and risk can be estimated. It should not be too broad because then the effects, causes and risk can not be estimated, and it should not be too narrow because in that case it is not describing a major system function.

In the second stage, an assessment of criticality on the identified functional failure modes is performed by means of an FMEA. The goal of the analysis on the system level is to determine the most critical system functions of the system as a whole. A criticality analysis on this level is required to focus the remaining part of the failure analysis only to those functions that have a major effect on a system failure. The starting point for the FMEA is the fault tree obtained from the FTA. With this information, the first three columns of an FMEA sheet (refer to Fig. 1) can be completed. Subsequently, for each failure mode, the possible local effects, end effects, causes and methods of detection are evaluated. Next, the analyst can determine a severity index $(S)$, occurrence index $(O)$ and detectability index $(D)$ for each failure mode to calculate an RPN (where $R P N=S \cdot O \cdot D$ ), see Section 2.1. This results in a rather detailed risk assessment with a quantitative judgement and it provides information on how critical each functional failure mode is. Next, these failure modes can be ranked according to RPN to select the functional failure mode(s) that are the most critical and should be analysed further. As our method is explicitly based on limiting the depth of the FTA in each level, we consider such an analysis to be complete, so that using the diamond event for the failure mode(s) that are not analysed further does not add much value.

How many failure modes are selected for further analysis depends on the time available for the complete analysis and on the goal of the analysis. We propose to determine an RPN cut-off value $C_{\text {system }}$ to select the functional failure modes for further analysis. $C_{\text {system }}$ is then the decision variable that can be used to balance between the required time for the analysis and the extend of the analysis. If $C_{\text {system }}$ is lowered, more failure modes are considered and the analysis will be broader, but will take more time, and vice versa. It is recommended to initially set $C_{\text {system }}$ not too low to prevent a too comprehensive analysis.

\subsubsection{Function level}

Next, the analysis shifts one level down to the function level. Again, an FTA is performed for identification of failure modes and an FMEA for assessment of the criticality. The analysis on function level is performed on each of the selected functional failure modes at the previous level (determined by the value of $C_{\text {system }}$ ).

In the first stage, the FTA, the top event is a failure of a system function (a function failure mode). The scope of the analysis is the system function and the depth of analysis is down to component failures. Thus, the FTA is sufficient when component failure modes are identified. Subsequently, in the second stage analysis, an FMEA is performed to assess the criticality of each component failure mode. As in the system level analysis, the component functional failure modes can now be ranked according to RPN to identify the most critical failure modes. As for the system level analysis, it is proposed to use a cut-off value $C_{\text {function }}$ as a decision variable to select critical failure modes for further analysis. In comparison with $C_{\text {system }}$, $C_{\text {function }}$ is expected to be lower as typically elements lower in the system hierarchy (e.g., components) have a smaller effect on the system reliability than higher level elements (e.g., subsystems). This effect also decreases the criticality of the elements lower in the system hierarchy. Based on a case study (see Section 4), a pragmatic guideline is to determine $C_{\text {function }}$ in such a way that, for example, no more than $50 \%$ of the component failure modes are selected for further analysis. But again, the actual value of $C$ is determined by available time and the objective of the analysis.

The goal of the second stage at this level is to link a critical system functional failure to component failures and assess its impact through a criticality assessment. As such, this analysis provides tangible infor- 
mation on which component failures affect a major system functional failure that may result in a system failure.

\subsubsection{Component level}

In this third level, the analysis shifts to the deepest level in a complex engineering system: the component level. As in the previous analyses, both an FTA and FMEA are performed. In the first stage, the FTA, the top event is a component failure (a component failure mode). The scope in this FTA is narrowed to the component itself and the depth of analysis is to the level of failure mechanisms. Failure mechanisms are the deepest level of failures that are considered in this paper and describe the physical mechanisms underlying the failures of parts, components or structures [2], like for example fatigue, wear or overload. This means that the real root cause of the failure is assessed. The second stage analysis at this level then comprises an FMEA of the identified failure mechanisms. Formally this cannot be called an FMEA anymore, as the analysis is now based on failure mechanisms rather than failure modes. However, the procedure is the same as on the previous levels: for each failure mechanism the RPN can be determined and the most critical failure mechanism(s) can be identified. As stated previously, the goal of this final level is to understand the underlying failure mechanisms of a component failure. This knowledge will be particularly valuable in two situations. Firstly, when a root cause analysis is performed to solve a recurring failure, knowledge on the failure mechanism (and governing loads) will assist in finding a suitable solution. This will always be either modifying the design to increase the capacity, or reducing the loads $[2,20,21]$. Secondly, when one wants to design a maintenance policy based on a component's condition (i.e., condition based maintenance), it is required to understand the determinants of this condition. Knowledge of the critical failure mechanism will then guide the selection of sensor type and location, and of the appropriate analysis technique.

\section{Case study: an additive manufacturing system}

In this section, a case study of the application of the recursive FTAFMEA methodology is discussed. First, in Section 4.1 the system under study is described. Second, Section 4.2 discusses the results of the application of the failure analysis. Because of confidentiality and simplification of the discussion, we only show key parts of the results to illustrate the methodology. We have used Microsoft Excel and Visio software to support and document the analysis results. In Section 4.3, we reflect on the results.

\subsection{Context}

The case study is performed in cooperation with a Dutch original equipment manufacturer of industrial additive manufacturing (AM) systems, Additive Industries [22]. The methodology is applied to their most recent metal additive manufacturing system, MetalFAB1. The MetalFAB1 is a modular AM system that consists of several complex mechatronic modules. It is shown in Fig. 4.

\subsection{Results}

In this section the results of the failure analysis are presented. The discussion is divided into three sections: system level (Section 4.2.1), function level (Section 4.2.2) and component level (Section 4.2.3).

\subsubsection{System level analysis}

First, a system level FTA is performed to identify the functional failure modes. A functional failure mode is defined as a failure of a major system function for which its effects, causes and risk can be estimated. The fault tree analysis on this level is sufficiently detailed as soon as all functional failure modes have been identified. The top event for the system level FTA is a failure of the system as a whole, which is in this case that the MetalFAB1 is not functioning according to the specification. A key part
Table 2

System level FMEA (partial).

\begin{tabular}{llllll}
\hline & Failure mode & S & O & D & RPN \\
\hline 1 & Exposure module handling failure & 5 & 2 & 1 & 10 \\
2 & Controls module process conditioning failure & 8 & 5 & 1 & 40 \\
3 & AM core module process conditioning failure & 8 & 5 & 1 & 40 \\
4 & Powder layer deposit failure & 8 & 5 & 3 & 120 \\
5 & Exposure failure & 8 & 5 & 3 & 120 \\
6 & Build storage failure & 5 & 2 & 1 & 10 \\
7 & Heat treatment failure & 5 & 8 & 1 & 40 \\
8 & Load/unload AM core module failure & 5 & 2 & 1 & 10 \\
9 & Load/unload storage module failure & 5 & 2 & 1 & 10 \\
10 & Load/unload heat treatment module failure & 5 & 2 & 1 & 10 \\
11 & Robot failure & 5 & 2 & 1 & 10 \\
12 & Load/unload exchange module failure & 5 & 2 & 1 & 10 \\
\hline
\end{tabular}

of the resulting fault tree is shown in Fig. 5; a number of branches is not depicted to simplify the diagram. It shows 12 functional failure modes that are identified. In order to find these functional failure modes in accordance with the definition, several intermediate events had to be created with FTA logic [5].

Subsequently, an FMEA is performed over the identified functional failure modes. Table 2 shows the resulting RPN values of this analysis. From these twelve functional failure modes, two have an RPN of 120 , three an RPN of 40 and seven an RPN of 10 . Notice that several columns of the FMEA-sheet (see Fig. 1) are omitted, since these are not relevant for this discussion. The cut-off value $C_{\text {system }}$ is used to select failure modes for further analysis. For instance, if $C_{\text {system }}=40$, then the top five functional failure modes are selected for further analysis: functional failure mode $2,3,4,5$ and 7 . These functional failure modes are then analysed further with a function level analysis (see Fig. 2).

\subsubsection{Function level analysis}

The selected critical functional failure modes are analysed further in a two-stage function level analysis. The first stage is the function level FTA, followed by a function level FMEA. Only functional failure mode powder layer deposit failure is analysed further in this case study.

First the function level FTA is performed. This results in the fault tree that is partly depicted in Fig. 6, in which the top event is the functional failure mode powder layer deposit failure. Subsequently, all intermediate events are identified that can cause this functional failure mode with FTA logic [5]. The analysis is sufficient when component failure modes have been identified. Fig. 6 shows 18 component failure modes that have been identified. A number of branches is not depicted to simplify the diagram.

Next, the second stage analysis is performed. Similar to the system level FMEA (Section 4.2.1), a function level FMEA is performed over the identified functional failure modes to prioritize the failure modes. The results are shown in Table 3 . The cut-off value $C_{\text {function }}$ is used to determine which failure modes should be analysed further. In Table 4, an analysis on $C_{\text {function }}$ in relation to the selected critical component failure modes is shown. It shows that, if $32 \leq C_{\text {function }} \leq 200$ the number of selected component failure modes is relatively low, so only the most critical failures are selected. If $C_{\text {function }}$ is chosen lower than 32 , for instance $C_{\text {function }}=16$, then almost all components are selected for further analysis (89\%). As a result, the efficiency gain of using the proposed recursive FTA-FMEA methodology instead of a traditional complete FTA would be low. This observation was also found in other function level analyses of the MetalFAB1 system (not included in this discussion). We suggest a pragmatic guideline to set $C_{\text {function }}$ in such a way that no more than $50 \%$ of the total component failure modes are selected. This ensures that the complete analysis remains focused on the most critical failures and that there is an efficiency gain with respect to a traditional (full) FTA. We advise to consider this guideline as a pragmatic rule of thumb, which may be neglected under certain circumstances (e.g., when a specific important function should be analysed thoroughly). More re- 


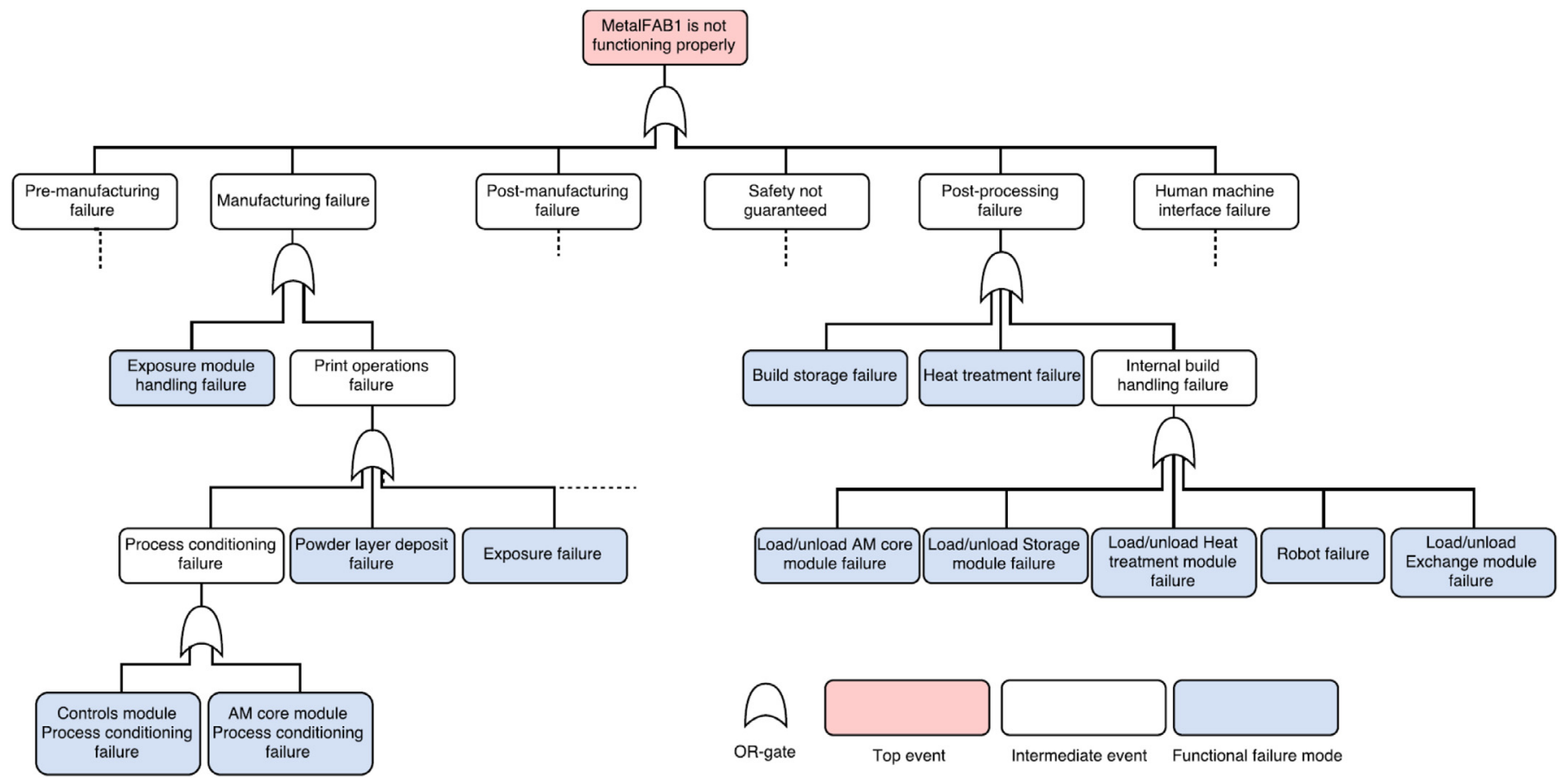

Fig. 5. System level FTA (partial).

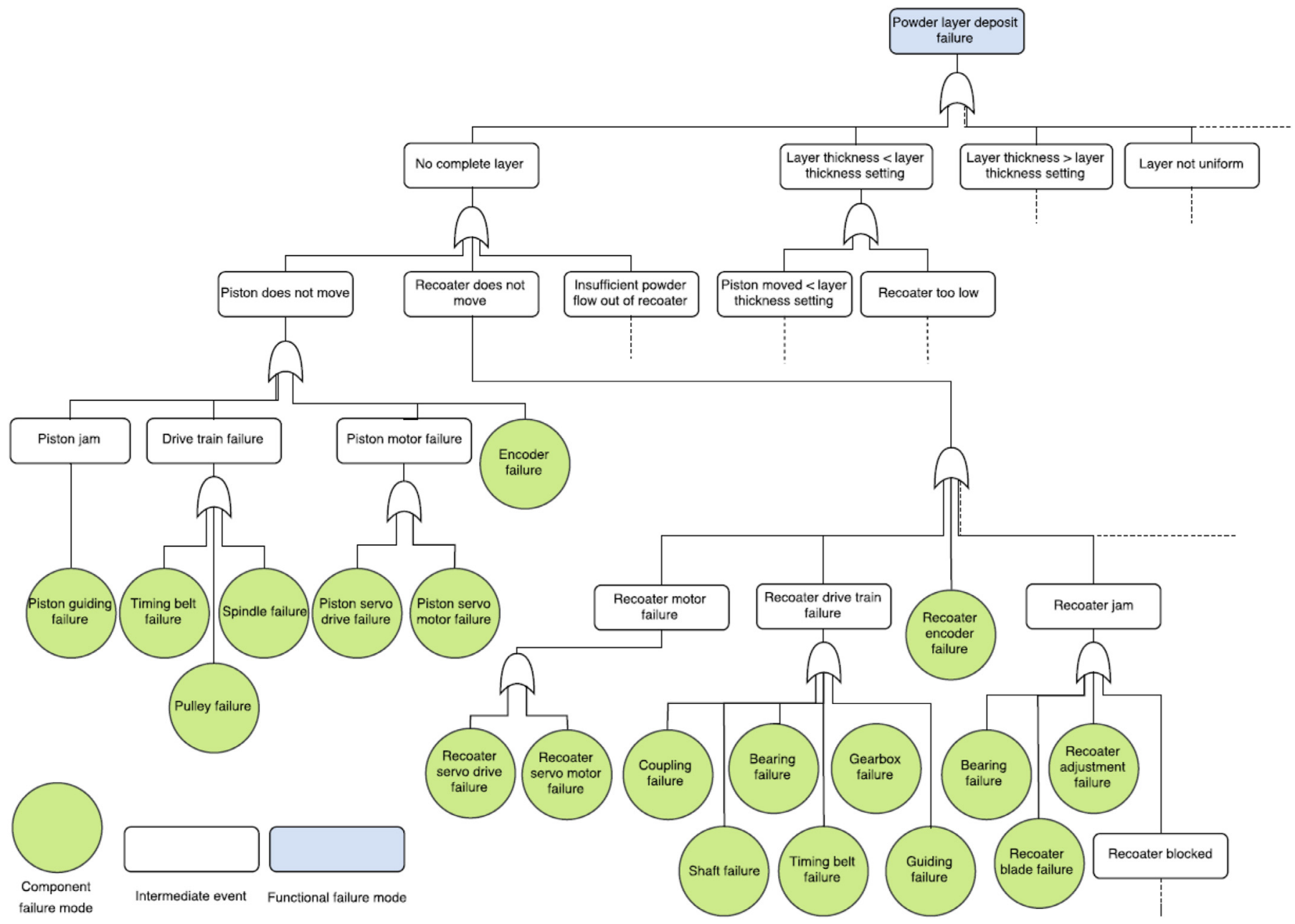

Fig. 6. Function level FTA (partial). 
Table 3

Function level FMEA (partial).

\begin{tabular}{llllll}
\hline & Failure mode & S & O & D & RPN \\
\hline 1 & Piston guiding failure & 8 & 2 & 5 & 80 \\
2 & Pulley failure & 8 & 1 & 2 & 16 \\
3 & Spindle failure & 8 & 1 & 5 & 40 \\
4 & Piston timing belt failure & 8 & 1 & 2 & 16 \\
5 & Piston servo motor failure & 8 & 1 & 2 & 16 \\
6 & Piston servo drive failure & 8 & 1 & 2 & 16 \\
7 & Piston encoder failure & 8 & 1 & 5 & 40 \\
8 & Recoater servo motor failure & 8 & 1 & 2 & 16 \\
9 & Recoater servo drive failure & 8 & 1 & 2 & 16 \\
10 & Recoater encoder failure & 8 & 1 & 2 & 16 \\
11 & Coupling failure & 8 & 1 & 2 & 16 \\
12 & Shaft failure & 8 & 1 & 2 & 16 \\
13 & Bearing failure & 8 & 2 & 2 & 32 \\
14 & Recoater timing belt failure & 8 & 1 & 1 & 8 \\
15 & Recoater gearbox failure & 8 & 1 & 2 & 16 \\
16 & Recoater guiding failure & 8 & 2 & 5 & 80 \\
17 & Recoater blade failure & 8 & 5 & 5 & 200 \\
18 & Recoater adjustment & 8 & 1 & 1 & 8 \\
\hline
\end{tabular}

Table 4

Comparison of $C_{\text {function }}$.

\begin{tabular}{llll}
\hline$C_{\text {function }}$ & $\begin{array}{l}\text { Total component } \\
\text { failure modes }\end{array}$ & $\begin{array}{l}\text { Selected component } \\
\text { failure modes }\end{array}$ & $\%$ \\
\hline 200 & 18 & 1 & $6 \%$ \\
80 & 18 & 3 & $17 \%$ \\
40 & 18 & 5 & $28 \%$ \\
32 & 18 & 6 & $33 \%$ \\
16 & 18 & 16 & $89 \%$ \\
\hline
\end{tabular}

Table 5

Component FMEA (partial).

\begin{tabular}{llllll}
\hline & Failure mode & S & O & D & RPN \\
\hline 1 & Thermal degradation & 8 & 2 & 2 & 64 \\
2 & Wear of recoater guiding & 8 & 5 & 5 & 200 \\
3 & Contamination & 8 & 2 & 1 & 16 \\
4 & Wear of recoater carriage & 8 & 5 & 5 & 200 \\
5 & Overload of recoater blade & 8 & 8 & 1 & 64 \\
6 & Wear of recoater blade & 8 & 8 & 5 & 320 \\
\hline
\end{tabular}

search is recommended to improve the definition of the optimal cut-off value.

\subsubsection{Component level analysis}

In this section, the third and deepest level of analysis is discussed, the component level. Similar to the previous analyses, this is a two-stage analysis. To show the idea of the analysis, the component failure mode recoater blade failure is investigated.

First, the component level FTA is performed to identify the underlying failure mechanisms. The top event is a component failure mode, in this case recoater blade failure. Similar to the system level and function level FTA, the FTA logic is used to identify all intermediate events that can cause this component failure mode. The analysis is sufficient when failure mechanisms have been identified. The key part of the fault tree is depicted in Fig. 7, in which six failure mechanisms are shown. A number of branches is not depicted to simplify the diagram.

The analysis is completed with a component level FMEA (formally not an FMEA, see Section 3.3.3). The result is depicted in Table 5. These results now provide detailed information on the most critical failures in the system.

\subsection{Reflection}

The case study gives the following insights. First, the results of the analysis help Additive Industries' engineers to better understand the fail-

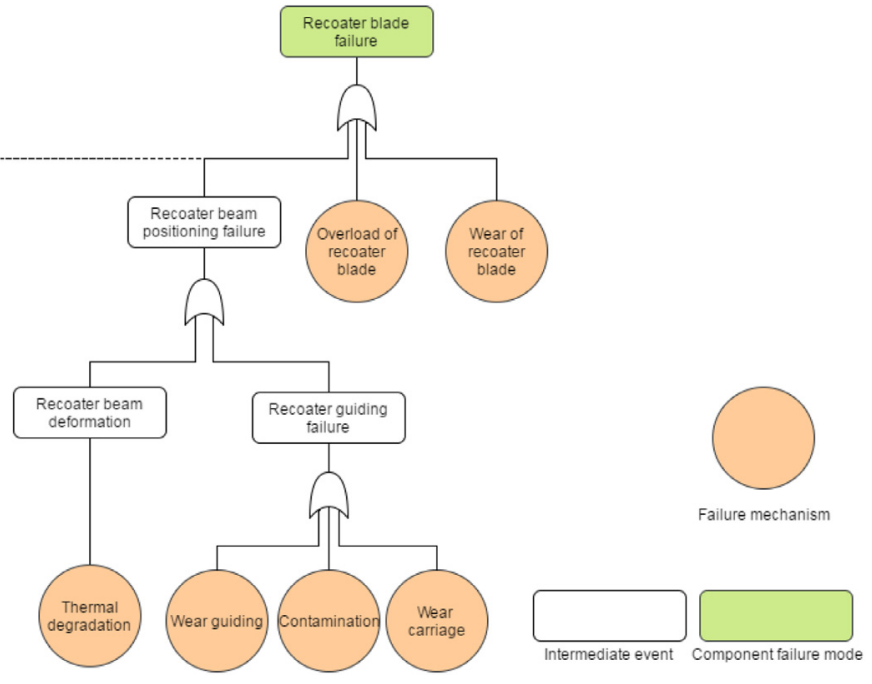

Fig. 7. Component level FTA (partial).

ure behaviour of the MetalFAB1 system, which they can use to improve the system design. Since the recursive FTA-FMEA approach leads to information down to the level of failure mechanisms, the engineers have profound directions for improvement. For instance, redesign of parts of the system is considered to eliminate or reduce the risk of critical failure modes knowing that they are caused by specific failure mechanisms. Second, the overview of identified failure modes of the system is used by the service and maintenance organisation to develop a (preventive) maintenance programme. For instance, the high risk for certain failure modes is reduced by performing appropriate preventive maintenance actions (e.g., regular inspections and/or preventive replacement).

The company was satisfied with the results of the case study and recognizes the benefits of the new method compared to a traditional FMEA or FTA. The Lead of Mechanical Engineering at Additive Industries stated that: "The proposed recursive FMEA-FTA method has been selected and successfully implemented by Additive Industries to improve the insight in the failure behaviour of the MetalFAB1 system, while the resource claim for a full traditional FMEA or FTA has been rejected based on a cost-benefit analysis."

We believe that the method would lead to similar results in other environments where equipment is built with a modular architecture, which is very common in many industries nowadays. Equipment with a highly integrated architecture is often a simple system. But in the case of a more complex system with an integrated architecture, the efficiency gain of our proposed method could be limited compared with a traditional FTA, since many functions will be represented by the same components. On the other hand, the proposed method could help the analysts to work in a more structured way.

\section{Conclusion}

In this paper, we have proposed a method to perform failure analysis. Fault tree analysis (FTA) and failure modes and effect analysis (FMEA) are two existing methods, that can also be combined. However, the main drawback of applying these methods is that it is time consuming. As a result, the methods are often not applied thoroughly, which can lead to not identifying important failure modes.

The method that we have proposed applies FTA and FMEA in a recursive manner, thus building on proven, existing methods. The key benefits of our method are that it is well structured, does not take too much time (i.e., is efficient), while it does enable its users to find all relevant failure modes (i.e., is effective). A traditional FMEA of a complex system, for instance a large diesel engine or wind turbine, takes typically 
a few weeks to complete. However, this highly depends on the people involved (number, skills and knowledge), the number of critical parts in the system, the required level of detail of the analysis and the skills of the FMEA facilitator. The recursive FTA-FMEA framework increases the efficiency in the following ways. First, the complete analysis is decoupled into sub analyses that each have a clear scope and required level of detail. Second, between the analyses on system level, function level and component level a priority selection step is proposed. This includes the selection of failure modes that should be analysed further based on an RPN cut-off value.

An application to an industrial additive manufacturing system shows how a company can benefit from the new failure analysis methodology.

The proposed method can be extended as follows. First, in Section 3.1 it is argued that complex machinery typically has a layered architecture. In this paper, three levels have been identified (system, functions and components), leading to a decoupling of the failure analysis in three levels. An extension of the proposed methodology could be to decouple between more than three levels. For example, in a complex system one may identify the level of (sub-)modules in between functions and components. It depends on the size of the complete system whether decoupling between more levels makes sense. A disadvantage is that the number of analyses to perform increases the complexity and reduces the efficiency: for example, if one distinguishes five levels, more separate analyses have to be formed. Second, more research is recommended on the definition of the optimal cut-off value to select the most critical failure modes for further analysis in between the different levels of analysis. In this paper, a pragmatic rule of thumb is introduced to guide this decision (see Section 4.2.2), but a more extensive guideline could improve the effectiveness of the proposed FTA-FMEA methodology further.

\section{Acknowledgements}

The authors are grateful to Additive Industries for supplying a case study. We specifically thank Mark Vaes and Daan Kersten. The authors further acknowledge the support of the Netherlands Organisation for Scientific Research (NWO) under project number 438-13-207. Finally, the authors thank the three reviewers and the editor for their helpful comments.

\section{References}

[1] Bertsche B. Reliability in automotive and mechanical engineering: determination of component and system reliability. Springer Berlin Heidelberg; 2008 doi:10.1007/978-3-540-34282-3.

[2] Tinga T. Principles of loads and failure mechanisms: applications in maintenance, reliability and design. Springer London; 2013. doi:10.1007/978-1-4471-4917-0.

[3] DoD U. MIL-STD 1629A failure modes, effects, and criticality analysis. 1980.

[4] Blanchard B, Fabrycky W. Systems engineering and analysis. Springer; 2006.

[5] Stamatelatos M, Vesely W, Dugan J, Fragola J, III JM, Railsback J. Fault tree handbook with aerospace applications. NASA; 2002.

[6] Hamada MS, Wilson AG, Rees CS, Martz HF. Bayesian reliability. Springer New York; 2008. doi:10.1007/978-0-387-77950-8

[7] Ruijters E, Stoelinga M. Fault tree analysis: a survey of the state-of-the-art in modeling, analysis and tools. Comput Sci Rev 2015;15:29-62

[8] Yu S, Liu J, Yang Q, Pan M. A comparison of fmea, afmea and fta. In: Reliability, maintainability and safety (ICRMS), 2011 9th international conference on; 2011. p. 954-60. doi:10.1109/ICRMS.2011.5979423.

[9] Bluvband Z, Polak R, Grabov P. Bouncing failure analysis (bfa): the unified fta-fmea methodology. In: Annual reliability and maintainability symposium, 2005. Proceedings.; 2005. p. 463-7. doi:10.1109/RAMS.2005.1408406.

[10] Han X, Zhang J. A combined analysis method of fmea and fta for improving the safety analysis quality of safety-critical software. In: Granular computing (GrC), 2013 IEEE international conference on; 2013. p. 353-6. doi:10.1109/GrC.2013.6740435.

[11] Moubray J. The case against streamlined reliability centered maintenance. Maint Asset Manag 2001;16:15-27.

[12] Blanchard B, Fabrycky W. Reliability-centered maintenance guide: for facilities and collateral equipment. NASA; 2008.

[13] Nowlan F, Heap H. Reliability-centered maintenance. United Airlines; 1978. Reproduced by US Departement of Commerce Springfied.

[14] Selvik JT, Aven T. A framework for reliability and risk centered maintenance. Reliab Eng Syst Saf 2011;96(2):324-31.

[15] Liu H-C, Liu L, Liu N. Risk evaluation approaches in failure mode and effects analysis: a literature review. Expert Syst Appl 2013;40(2):828-38.

[16] Xiao N, Huang H-Z, Li Y, He L, Jin T. Multiple failure modes analysis and weighted risk priority number evaluation in FMEA. Eng Failure Anal 2011;18(4):1162-70. doi:10.1016/j.engfailanal.2011.02.004.

[17] Wang Y-M, Chin K-S, Poon GKK, Yang J-B. Risk evaluation in failure mode and effects analysis using fuzzy weighted geometric mean. Expert Syst Appl 2009;36(2, Part 1):1195-207. doi:10.1016/j.eswa.2007.11.028.

[18] Waeyenbergh G. Cibocof. a framework for industrial maintenance concept development, Ph.D. thesis. KU Leuven; 2005.

[19] Brunelli M. Introduction to the analytic hierarchy process. Springer International Publishing; 2015. doi:10.1007/978-3-319-12502-2.

[20] Tinga T. Mechanism based failure analysis. Improving maintenance by understanding the failure mechanisms. Netherlands Defence Academy; 2012. URL http://doc.utwente.nl/83622/.

[21] Tinga T. Application of physical failure models to enable usage and load based maintenance. Reliab Eng Syst Saf 2010;95(10):1061-75. doi:10.1016/j.ress.2010.04.015.

[22] Additive Industries BV. Additive industries corporate website. 2017. URLhttp://additiveindustries.com/Home. 